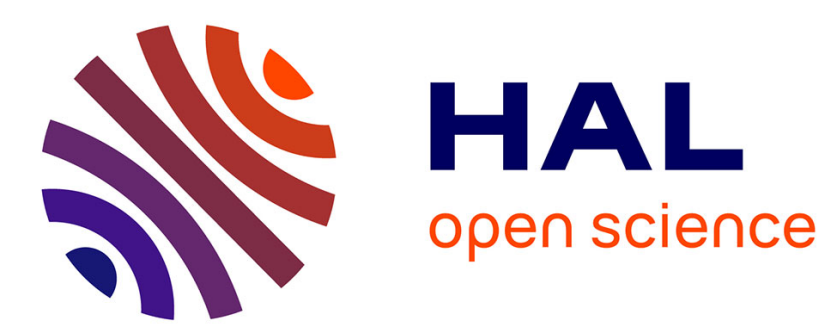

\title{
Nouvel examen de l'armature composite magdalénienne du Tuc d'Audoubert (Montesquieu-Avantès, Ariège) Jean-Marc Pétillon, Pierre Cattelain
}

\section{To cite this version:}

Jean-Marc Pétillon, Pierre Cattelain. Nouvel examen de l'armature composite magdalénienne du Tuc d'Audoubert (Montesquieu-Avantès, Ariège). Bulletin de la Société préhistorique française, 2004, 101

(1), pp.45-53. halshs-00403690

\section{HAL Id: halshs-00403690 \\ https://shs.hal.science/halshs-00403690}

Submitted on 12 Jul 2009

HAL is a multi-disciplinary open access archive for the deposit and dissemination of scientific research documents, whether they are published or not. The documents may come from teaching and research institutions in France or abroad, or from public or private research centers.
L'archive ouverte pluridisciplinaire HAL, est destinée au dépôt et à la diffusion de documents scientifiques de niveau recherche, publiés ou non, émanant des établissements d'enseignement et de recherche français ou étrangers, des laboratoires publics ou privés. 
Citer comme :

PÉTILLON J.-M., CATTELAIN P. (2004) - Nouvel examen de l'armature composite magdalénienne du Tuc d'Audoubert (Montesquieu-Avantès, Ariège). Bulletin de la Société préhistorique française, 101 (1), p. 45-53.

\title{
Nouvel examen de l'armature composite magdalénienne du Tuc d'Audoubert (Montesquieu-Avantès, Ariège)
}

\author{
Jean-Marc PÉTILLON et Pierre CATTELAIN
}

\begin{abstract}
Résumé
Nous exposons ici les résultats de l'étude de deux pièces d'industrie osseuse - une pointe à base fourchue et une pré-hampe, toutes deux en bois de renne - découvertes au début du $\mathrm{XX}^{\mathrm{e}}$ siècle dans l'habitat magdalénien de la grotte du Tuc d'Audoubert. Ces objets ont la particularité d'avoir été interprétés à l'époque comme les deux constituants d'une armature de projectile composite. Leur réexamen a permis de confirmer cette attribution fonctionnelle, de montrer la proximité typotechnologique de la pointe à base fourchue du Tuc d'Audoubert avec ses homologues des grottes d'Isturitz, Gourdan et Lortet, et enfin de rapprocher la préhampe du Tuc d'Audoubert de pièces similaires identifiées au sein des séries d'Isturitz. Les implications fonctionnelles de ce type d'armature composite - hypothèses sur le rôle de la pré-hampe, nature probable du projectile qui lui est associé - sont développées en conclusion.
\end{abstract}

\begin{abstract}
In 1926, H. Bégouën reported the find of a $68 \mathrm{~mm}$ long fork-based antler projectile point and a $344 \mathrm{~mm}$ long fragmentary antler foreshaft in the Magdalenian dwelling of the Tuc d'Audoubert cave site. Since both objects fit perfectly into each other, the author recognised them at the time as the two components of a composite projectile tip. Re-examination of these artifacts has allowed us to confirm this functional interpretation, and to demonstrate the typological and technological relationship of the Tuc d'Audoubert fork-based point with similar points from the Isturitz, Gourdan and Lortet caves. As for the Tuc d'Audoubert foreshaft, it can be compared with several similar items from the Isturitz series. Our conclusion discusses the functional implications of this composite weapon tip: the possible role of the foreshaft, and probable nature of the associated projectile (i.e. spear or arrow).
\end{abstract}

Mots-clefs : Magdalénien, Tuc d'Audoubert, industrie osseuse, pointes à base fourchue, préhampe, sagaie.

Keywords : Magdalenian, Tuc d'Audoubert, osseous industry, fork-based points, foreshaft, spear.

En 1926, le comte H. Bégouën publiait la description de deux objets magdaléniens en matière osseuse, découverts à proximité l'un de l'autre dans la grotte du Tuc d'Audoubert. Il s'agissait d'une longue baguette façonnée interprétée comme une "préhampe", ou élément intermédiaire de hampe de projectile, et d'une pointe de sagaie à base fourchue "s'adaptant admirablement au bout (...) de cette baguette" (Bégouën, 1926, p. 224). De cette trouvaille, l'auteur déduisait l'existence au Magdalénien d'armatures composites, constituées de deux pièces osseuses emboîtées l'une dans l'autre : "il semble donc que ces pointes à base fourchue ne se fixaient pas directement sur un manche en bois, mais qu'un os fixé nous ne savons comment servait d'intermédiaire entre la pointe et la hampe de la lance" (ibid.). 
Ces deux pièces sont conservées dans les collections du Département de Préhistoire du Musée de l'Homme et C. Leroy-Prost eut l'amabilité de nous en proposer l'étude début 2002. Elles avaient en effet déjà été incluses dans la fiche typologique des éléments intermédiaires de hampe de projectile (Cattelain, 1993), mais sans que l'auteur ait eu à l'époque l'occasion de les réexaminer. Par ailleurs, dans le cadre d'un mémoire universitaire, l'un d'entre nous (Pétillon, 2000a) avait analysé une série de 549 pointes à base fourchue provenant des sites d'Isturitz (Saint-Martin-d'Arberoue, PyrénéesAtlantiques), Gourdan (Gourdan-Polignan, Haute-Garonne) et Lortet (Lortet, Haute-Garonne). Cette étude avait permis d'apporter des résultats nouveaux sur l'emmanchement de ce type d'armature, concernant notamment la question des préhampes. Il paraissait donc intéressant de reconsidérer les pièces du Tuc d'Audoubert à la lumière de ce nouvel éclairage.

\section{Conditions de découverte et contexte archéologique}

La grotte du Tuc d'Audoubert est située dans les pré-Pyrénées ariégeoises. Avec Enlène et les TroisFrères, elle constitue l'ensemble des cavernes du Volp.

Le réseau hydrographique creusé par le Volp se développe sur trois niveaux : le niveau inférieur actif, le niveau médian partiellement inondable et le niveau supérieur, fossile. La grotte du Tuc d'Audoubert s'étend pour partie au niveau médian (Salle Nuptiale et galeries adjacentes) et pour partie au niveau supérieur (Galerie Supérieure). La caverne n'est aujourd'hui accessible que par bateau, en empruntant le niveau inférieur qui correspond au cours actuel du Volp (fig. 1).

Découvert en 1912 par H. Bégouën et ses trois fils, le Tuc d'Audoubert est surtout connu pour ses nombreuses représentations pariétales, ainsi que pour les deux bisons modelés en argile et les empreintes de pas humains qui se trouvent au fond de la Galerie Supérieure. Mais il existe également dans cette grotte un habitat paléolithique, situé dans une galerie du niveau médian communiquant avec le cours du Volp (fig. 1). Ce gisement fut repéré et partiellement fouillé par H. Bégouën en 1913.

Selon H. Bégouën, la stratigraphie se composait d'alluvions sablo-argileuses au milieu desquelles se trouvaient "deux couches archéologiques parfois distinctes et parfois confondues, n'ayant jamais plus de 8 à 10 centimètres d'épaisseur et ne présentant parfois qu'une simple ligne charbonneuse" (op. cit., p. 221). Ces couches ont livré une faune "peu abondante" (bison, renne, cheval, ours), des silex "abondants", des morceaux d'ocre, deux bâtons percés ainsi qu'un "grand nombre" de pointes de sagaies et de baguettes demi-rondes, mais pas de harpons ni d'aiguilles. La publication d'H. Begouën est surtout consacrée à la description des objets de parure (12 dents perforées, 3 pendeloques en os et bois de cervidé, 1 rondelle percée et 2 contours découpés) et des objets d'art mobilier (18 plaquettes gravées, 2 rondes-bosses en bois de renne et 5 os gravés).

R. Begouën et J. Clottes ont attribué cette occupation au Magdalénien moyen, en s'appuyant sur la présence d'outillage osseux caractéristique et sur certains détails stylistiques des plaquettes gravées (Bégouën et Clottes, 1983, 1990). Une datation radiocarbone réalisée sur des esquilles osseuses provenant des déblais des fouilles de 1913 a livré la date de $14350 \pm 160$ BP (Gif 5867), soit 15900 - 14600 BC (IntCal 98).

C'est au sein de cet ensemble archéologique - ou plutôt à côté, comme on le verra - qu'ont été découverts les deux objets auxquels cette note est consacrée.

\section{La pointe à base fourchue (fig. 2)}

Il s'agit d'une pointe en bois de cervidé longue de $68 \mathrm{~mm}$, dont la partie proximale est constituée d'une fourche bifide. L'extrémité distale a été fracturée anciennement ; la facette de fracture est trop émoussée pour être encore lisible. La pièce est sinon en très bon état de conservation.

\section{Données morphométriques}

L'étude typologique des 549 pointes à base fourchue d'Isturitz, Gourdan et Lortet nous avait permis de mettre en lumière le caractère extrêmement normalisé de ce type d'armature, notamment en ce 
qui concerne les dimensions de la partie fourchue et le "calibre" des pointes - forme de la section, largeur et épaisseur maximales (Pétillon, 2000a, 2002). Cette impression de standardisation n'est pas démentie par la pointe à base fourchue du Tuc d'Audoubert.

Celle-ci est en effet de section quadrangulaire, à angles arrondis. Sa largeur et son épaisseur maximales, mesurées au niveau de la naissance des fourchons, sont respectivement de 9 et $7 \mathrm{~mm}$. La longueur de sa partie fourchue est de $32 \mathrm{~mm}$; à l'extrémité proximale de la pièce, l'écartement entre les deux fourchons est de 5,5 $\mathrm{mm}$. Or, ces mesures placent la pointe du Tuc d'Audoubert dans la moyenne des dimensions relevées sur les bases fourchues d'Isturitz, Gourdan et Lortet (tabl. 1 et fig. 3) ; seul l'écartement des fourchons est supérieur à la moyenne, tout en restant à l'intérieur de l'amplitude de variation constatée sur nos trois sites de référence (entre 3 et $6 \mathrm{~mm}$ pour $78 \%$ des pièces). La section quadrangulaire est également un caractère typique des pointes à base fourchue, puisqu'elle est présente sur plus de $72 \%$ des pièces d'Isturitz, Gourdan et Lortet.

Au sein de notre corpus de référence, parmi les pointes à base fourchue de première facture - c'està-dire celles qui ne présentent pas de traces de réaffûtage de l'extrémité distale - nous avions distingué deux sous-populations en ce qui concerne la longueur de la partie mésio-distale : les pointes longues (longueur mésio-distale généralement comprise entre 75 et $115 \mathrm{~mm}$ ) et les pointes courtes (longueur mésio-distale généralement comprise entre 35 et $65 \mathrm{~mm}$ ). La pointe du Tuc d'Audoubert est certes fracturée à l'extrémité distale, mais étant donné la faible épaisseur du fût au niveau de la fracture, on peut penser que la portion manquante ne représente pas plus de 10 à $15 \mathrm{~mm}$ au maximum. Lorsque cette pointe était complète, sa partie mésio-distale, qui mesure actuellement $36 \mathrm{~mm}$, ne devait donc pas dépasser les $50 \mathrm{~mm}$ - ce qui place cette pièce dans la catégorie des pointes courtes (1).

\section{Données techniques}

La pointe a été fabriquée dans la partie compacte d'un bois de cervidé ; sa face inférieure porte encore des traces de tissu spongieux, sur $1 \mathrm{~mm}$ d'épaisseur au maximum (à mi-hauteur de la pièce). Cette spongiosa est organisée en alvéoles étroits et serrés, caractéristiques du bois de renne. L'épaisseur importante du tissu compact $(6 \mathrm{~mm})$ nous permet d'avancer que la pointe a dû être débitée sur un bois de renne mâle adulte, probablement sur la perche ( $c f$. Averbouh, 2000, p. 99).

La pièce est entièrement façonnée, et sa surface ne porte plus aucune trace qui pourrait nous renseigner sur les techniques utilisées lors du débitage. Par analogie avec les données fournies par notre corpus de référence, on peut supposer que cette pointe a dû être fabriquée à partir d'un support de type "baguette en tasseau", débitée au moyen d'un rainurage longitudinal parallèle.

La pointe porte sur ses quatre faces des stries fines, régulières et continues, qui indiquent un façonnage par raclage longitudinal. En partie proximale, sur les faces externes des fourchons, on observe une série de stries plus marquées, qui se superposent aux stries de façonnage susmentionnées ; elles témoignent d'un raclage plus prononcé en cet endroit, opération sur l'interprétation de laquelle nous reviendrons plus loin.

La fourche elle-même a été façonnée au moyen d'un rainurage symétrique opposé, effectué à partir des faces supérieure et inférieure, et poursuivi jusqu'à ce que les deux rainures se rejoignent pour former un évidement. Les stigmates laissés par cette opération sont visibles sur les faces internes des fourchons, sous la forme de stries partant des faces inférieure et supérieure de la pointe et convergeant vers l'intérieur. Des stigmates identiques ont été obtenus sur des pointes à base fourchue expérimentales en bois de renne, pour lesquelles le rainurage de la fourche avait été effectué avec un burin dièdre (Pétillon, sous presse).

\section{La pièce interprétée comme pré-hampe (fig. 4)}

\section{Description et données morphométriques}

Cet objet est une baguette en matière dure animale entièrement façonnée, en très bon état de conservation. D'une longueur déjà impressionnante $(344 \mathrm{~mm})$, elle est de surcroît incomplète puisque ses deux extrémités sont fracturées ( $c f$. ci-dessous). Du côté le plus large et le plus épais, 
que nous désignerons comme proximal, la baguette est de section pratiquement carrée (14 $\mathrm{x}$ $13 \mathrm{~mm}$ ), aux angles très arrondis. D'une rectitude et d'une régularité parfaites, elle va ensuite en s'amincissant et s'aplatissant progressivement, se réduisant à une largeur de $7 \mathrm{~mm}$ et une épaisseur de $9 \mathrm{~mm}$ juste avant l'extrémité distale. Celle-ci présente un aménagement particulier que nous détaillerons plus bas. Enfin, du côté proximal, la pièce est décorée d'un motif constitué de deux incisions longitudinales subparallèles, reliées par sept courtes incisions transversales, comme les barreaux d'une échelle. Ce motif large de 2 à $3 \mathrm{~mm}$ se retrouve exactement à l'identique sur chacune des deux faces latérales de l'objet; il s'étend sur une longueur de 25 à $30 \mathrm{~mm}$, avant d'être interrompu par la fracture proximale.

\section{Données techniques}

Contrairement à ce que considérait $\mathrm{H}$. Bégouën ( $o p$. cit.), cette pièce n'est pas "taillée dans un os long de proboscidien, mammouth ou rhinocéros" : elle est en bois de cervidé, et presque totalement constituée de tissu compact. Des traces de spongiosa sont visibles sur la face inférieure, mais sur une épaisseur très faible, et uniquement sur une longueur d'environ $100 \mathrm{~mm}$, du côté de l'extrémité distale. La partie proximale, la plus massive, est entièrement compacte.

Un tel objet n'a pu être débité que sur la perche d'un bois de renne mâle adulte, présentant de surcroît une compacta particulièrement épaisse. La partie proximale de la pièce devait correspondre à la partie basilaire de la perche, où le tissu compact est le plus important (N. Goutas, com. pers.). Le façonnage, intégral, a fait disparaître toute trace de la surface anatomique d'origine, ainsi que les stigmates du débitage. Les traces de façonnage prennent la forme de stries de raclage longitudinal, sur les quatre faces de la pièce.

\section{Attribution fonctionnelle}

Comme on l'a vu, H. Bégouën proposait de faire de cette pièce une préhampe, c'est-à-dire un élément d'armature composite venant s'intercaler entre la hampe d'un projectile et la pointe proprement dite. Ce type d'objet doit donc "présent[er] à chacune de ses extrémités un dispositif d'emmanchement : biseau simple, biseau double ou fourche" (Cattelain, 1993, p. 15) (2). Qu'en estil de la pièce du Tuc d'Audoubert?

La partie distale présente sur une longueur de $25 \mathrm{~mm}$ un aménagement que H. Bégouën décrivait comme un "bout taillé en biseau et en gouge" (op. cit.). Cette morphologie est le résultat d'un rainurage symétrique opposé, dont les stries sont clairement visibles, effectué à partir des faces gauche et droite de l'objet et analogue à la technique utilisée pour la fabrication des bases fourchues (cf. ci-dessus). Mais ici le rainurage n'a pas été poursuivi jusqu'à créer un évidement : même si les rainures sont bien marquées et si les bords gauche et droit ont à cet endroit un profil nettement concave, il reste encore entre eux une épaisseur de 2 à $3 \mathrm{~mm}$ de matière osseuse. Il semble cependant que l'extrémité distale de l'objet se terminait bien à l'origine en une fourche bifide : on distingue en effet deux petites facettes de fracture, de $2 \mathrm{~mm}^{2}$ chacune environ, de part et d'autre de la rainure axiale. Ces facettes sont relativement nettes, et d'aspect plus frais que le reste de la pièce, indiquant une fracture postdépositionnelle. Elles constituent l'amorce de deux fourchons, qui vu leur faible épaisseur ne pouvaient qu'être de longueur réduite, 5 à $10 \mathrm{~mm}$ au maximum (fig. 5). Ce type d'aménagement fourchu a déjà été noté sur des pré-hampes du site d'Isturitz, et constitue à l'évidence un dispositif d'emmanchement ( $c f$. ci-dessous).

Du côté proximal, la pièce présente une fracture de grande ampleur, organisée en deux pans formant un angle aigu. A l'extrémité du pan droit se trouvent des traces en dents de scie évoquant un arrachement par flexion. Même si dans ce domaine les référentiels expérimentaux font en grande partie défaut, on peut déjà avancer que cette fracture n'est pas d'origine taphonomique ou postfouille, mais est survenue durant l'utilisation de l'objet. Si un dispositif d'emmanchement était présent à cet endroit, il a donc aujourd'hui disparu avec le fragment manquant. On peut toutefois supposer que, cette extrémité étant beaucoup plus large et plus épaisse que l'autre ( $c f$. mesures cidessus), il ne peut pas s'agir de l'extrémité distale fonctionnelle de l'objet. Pour des raisons de solidité de l'emmanchement tout comme de capacité de pénétration, il est en effet difficile 
d'imaginer une armature fixée sur une hampe par une fourche étroite $(7 \times 9 \mathrm{~mm})$, et qui s'évaserait ensuite pour atteindre un diamètre de $14 \mathrm{~mm}$ au niveau de la pointe. Même en l'absence de tout dispositif d'emmanchement, il est donc certain que le côté que nous avions désigné comme proximal est bien celui qui était en contact direct avec la hampe du projectile, et emmanché sur celle-ci.

La pièce du Tuc d'Audoubert devait donc bien posséder à l'origine un système d'emmanchement à chaque extrémité, ce qui correspond à la définition fonctionnelle de la préhampe.

\section{Association entre la pré-hampe et la pointe à base fourchue}

Selon H. Bégouën, la pointe à base fourchue et la préhampe sont associées et constituent les deux parties d'une même armature composite. Pour étayer son idée, il s'appuie sur quatre types d'arguments : les conditions de découverte des pièces, leurs caractères morphométriques, les comparaisons avec d'autres sites et les analogies ethnographiques.

\section{Conditions de découverte}

Les deux pièces n'ont pas été découvertes en connexion. Elles étaient cependant très proches l'une de l'autre : "à quelques centimètres en avant [de la préhampe] et de côté, nous avons trouvé dans le sol une pointe (...) à base fourchue" (Bégouën, op. cit.). De plus, ces objets étaient isolés et ne se trouvaient pas à l'intérieur des zones cendreuses qui ont livré le reste du matériel archéologique : "ces deux pièces n'ont pas été trouvées dans les foyers, mais un peu en avant, plus près de la rivière, dans les couches de sable argileux, comme si elles avaient été entraînées par les eaux, d'un gisement supérieur" (ibid.). Cette dernière hypothèse est indécidable en l'absence de données stratigraphiques plus précises ; mais quoi qu'il en soit, le fait que ces deux objets aient été découverts au même endroit et à l'écart des autres vestiges plaide plutôt en faveur de leur association.

\section{Morphométrie}

Les dimensions de la partie distale de la préhampe (largeur $7 \mathrm{~mm}$, épaisseur $9 \mathrm{~mm}$, section quadrangulaire) correspondent de très près à celles de la pointe (largeur $9 \mathrm{~mm}$, épaisseur $7 \mathrm{~mm}$, section quadrangulaire). H. Bégouën notait par ailleurs que les deux pièces pouvaient s'assembler étroitement, ce que nous avons pu vérifier grâce à un moulage réalisé par les services du Musée de l'Homme (fig. 6) : sans "forcer" aucunement l'écartement des fourchons, il est possible d'emboîter la pointe à l'extrémité de la préhampe, de telle sorte que l'ensemble présente une forme fuselée et régulière, presque sans solution de continuité. L'ajustement aurait été encore plus exact si l'extrémité distale de la pré-hampe avait été intacte, portant encore ses deux fourchons. Nous pouvons également interpréter maintenant les traces de raclage prononcées relevées sur les faces externes des fourchons de la pointe ( $c f$. ci-dessus) comme un moyen d'amincir l'extrémité desdits fourchons, de manière à adapter plus étroitement la pointe à la préhampe et d'affiner ainsi la silhouette générale de l'armature.

\section{Comparaisons avec d'autres sites}

H. Bégouën mentionne rapidement l'existence de pièces similaires à celles du Tuc d'Audoubert, provenant du Magdalénien de La Paloma (Soto de la Regueras, Asturias) et d'Isturitz. Aujourd'hui encore, ce sont effectivement les deux seuls gisements où ont été découvert des préhampes clairement associées à des pointes à base fourchue.

La préhampe de La Paloma possède deux extrémités en biseau double. Lorsqu'elle fut découverte en 1914-1915, l'une de ces extrémités était encore engagée entre les deux fourchons d'une pointe à base fourchue, le tout s'emboîtant si étroitement que les fouilleurs ont d'abord cru que la pièce était d'un seul tenant : "... en uno de los niveles del magdaleniense medio apareció la larga punta de flecha que representa la figura 16 ; al lavarla en el arroyo inmediato y limpiarla advertí que se descomponía en dos mitades que encajaban perfectamente una en otra..." (Hernández Pacheco, 1923, p. 23) (3). Pour autant qu'on puisse en juger d'après le dessin publié, ce biseau double possède 
des caractères métriques (longueur, largeur) proches de celles de l'extrémité distale de la pré-hampe du Tuc d'Audoubert.

En ce qui concerne les collections d'Isturitz, une grande partie des objets qui y étaient auparavant considérés comme préhampes ont été récemment réattribués. En effet, l'examen direct des pièces qui, sur la base des publications anciennes, avaient été inventoriées comme préhampes dans la fiche typologique (Cattelain, 1993), a montré que nombre d'entre elles étaient de "simples" pointes à base fourchue dont la forme de la partie distale avait été modifiée par réaffûtage (Pétillon, 2000b). En définitive, ce site n'a livré que trois pièces qui sont indiscutablement des éléments intermédiaires de hampe de projectile. L'un possède deux extrémités fourchues ; les deux autres présentent une extrémité fourchue opposée à une extrémité en biseau double. Par ailleurs, au sein des collections d'Isturitz, nous avons identifié 24 éléments fourchus fracturés comme des fragments de préhampes, de type fourche / biseau double, sur la base de comparaisons morphométriques avec les pièces entières. Nous avons montré que ces objets ont très probablement été utilisés de la manière suivante : leur extrémité à biseau double était emmanchée dans la hampe du projectile, tandis que leur extrémité distale fourchue venait s'emboîter dans la fourche, proximale, d'une pointe de sagaie (ibid.). Ce fonctionnement est similaire à celui proposé pour la pré-hampe du Tuc d'Audoubert. Dans les deux sites, les fourches situées à l'extrémité distale des préhampes présentent d'ailleurs des dimensions comparables (tabl. 2).

\section{Analogies ethnographiques}

H. Bégouën note que "cette coutume [l'insertion d'une préhampe] existe encore chez quelques peuplades sauvages. En Océanie, on insère souvent ainsi un os humain pour des raisons magiques" (Bégouën, op. cit.). L'ethnographie fournit effectivement de nombreux exemples d'armatures composites intégrant le principe de la préhampe, dont certaines utilisent des morphologies d'emmanchement proches de celles des pièces du Tuc d'Audoubert. A titre d'illustration, on peut ainsi citer les flèches des Îles de Santa Cruz évoquées par E. Passemard (1917) et décrites en détail par F. Speiser (1909) : certaines d'entre elles possèdent des armatures composées de plusieurs segments aux extrémités fourchues qui s'emboîtent les unes dans les autres. Si cet exemple ne constitue pas à proprement parler un argument en faveur de l'hypothèse $\mathrm{d}$ 'H. Begouën, il montre en tout cas que cette solution technique n'est pas une exclusivité du Magdalénien pyrénéo-cantabrique.

\section{Bilan}

En l'absence de stricte connexion archéologique, il n'est pas possible d'affirmer de façon catégorique que les pièces du Tuc d'Audoubert constituent bien les deux parties d'une même armature. Mais cette hypothèse peut tout de même être considérée comme très hautement probable, étant donné l'ensemble des éléments que nous avons évoqués - proximité spatiale des deux objets lors de leur découverte, étroite correspondance morphométrique entre la pointe et la préhampe, existence d'emmanchements similaires à Isturitz et La Paloma - qui tous accréditent cette idée.

\section{En guise de conclusion : quelques implications fonctionnelles}

\section{Conception des projectiles}

L'usage des préhampes a souvent été considéré par les préhistoriens comme un moyen de "réutiliser des pointes cassées, et donc forcément raccourcies, sans modifier les paramètres généraux du projectile (longueur, poids et équilibre, principalement)" (Cattelain, 1993, p. 21). Les pièces du Tuc d'Audoubert ne portant aucune trace de reprise ni de réparation, force est de constater que, comme nous le soulignions déjà (ibid.) et comme le montrent de nombreux projectiles ethnographiques, le système de l'armature composite peut également - voire le plus souvent - être intégré dans la conception initiale du projectile.

Reste alors le problème de l'utilité d'un tel dispositif. Sans qu'il soit possible d'exclure des motivations purement esthétiques ou culturelles, nous avions proposé une première série d'explications concernant l'usage des préhampes en bois de cervidé au Magdalénien : "la densité des 
matières dures animales fraîches est supérieure à celle des bois végétaux les plus répandus au Tardiglaciaire (pin, bouleau) et dans lesquels étaient probablement façonnées les hampes. De manière à équilibrer les projectiles en fonction de leur mode de lancer, il peut s'avérer utile d'allonger l'extrémité active en matière dure animale, ce qui alourdit la pointe. La présence de ces "rallonges" permet aussi de standardiser les longueurs de projectiles, en fonction des longueurs de bois brut disponibles" (ibid.). Les projectiles possédant des armatures composées de plusieurs segments sont peut-être également plus simples d'entretien : lorsqu'une fracture balistique endommage l'extrémité distale, ou que celle-ci se démanche lors d'un tir, elle peut être facilement remplacée sans avoir à reconfigurer l'ensemble de l'armature. Enfin, il est possible que la préhampe assure aussi une fonction de protection de la hampe lors des tirs, en absorbant une partie du choc de l'impact et en réduisant ainsi la fréquence des fractures au niveau du fût du projectile (Bergman, 1987). Toutes ces hypothèses demandent encore à être testées expérimentalement de manière systématique.

\section{Mode de lancer des projectiles}

En conclusion d'une étude d'ethnographie comparée portant entre autres sur les projectiles des peuples chasseurs actuels et subactuels, nous avions écrit que parmi les pointes paléolithiques, il n'était pas possible de distinguer les armatures de flèches des armatures de sagaie sur la base de simples critères morphométriques : "sauf cas extrêmes, la morphologie et la morphométrie de la plupart des pointes de projectile paléolithiques ne permettent pas de les classer, de facto, comme des pointes de sagaies (...) ou comme des pointes de flèches (...). La multiplicité des exemples [ethnographiques] montre bien que toute généralisation d'une "tendance" serait abusive. (...) Au propulseur, une pointe légère (...) est facilement compensée par une pré-hampe plus lourde et équilibrante ; à l'arc, une pointe quelque peu lourde peut être compensée par une longueur plus importante de la hampe : tout est une question d'équilibre et de choix techniques" (Cattelain, 1994, p. 20-21).

En revanche, le "calibre" des deux types de projectile est nettement différent - la plupart des flèches des chasseurs traditionnels possèdent une hampe dont le diamètre se situe en général autour de 8$9 \mathrm{~mm}$, tandis que les hampes de sagaies présentent systématiquement un diamètre très supérieur (13 à $14 \mathrm{~mm}$ pour la moyenne des sagaies australiennes).

On peut donc formuler l'hypothèse que, lorsqu'une armature paléolithique possède un diamètre très important au niveau de sa partie proximale, elle a probablement été emmanchée sur une hampe de fort calibre, évoquant plus une sagaie qu'une flèche. L'inverse n'est cependant pas vrai : si les dimensions de l'armature sont plus réduites, on ne peut rien conjecturer quant au type de projectile sur lequel elle était fixée... Une pointe de faible diamètre peut en effet très bien être emmanchée à l'extrémité d'une hampe lourde et épaisse, pour peu que celle-ci s'amincisse progressivement vers l'extrémité distale!

Ainsi, dans le cas qui nous occupe, la pointe à base fourchue du Tuc d'Audoubert présente des dimensions relativement réduites qui, si elle avait été découverte seule, auraient plutôt conduit à l'interpréter comme une pointe de flèche. Or, la préhampe à laquelle elle est associée possède une largeur et une épaisseur proximales telles qu'elle devait être fixée sur une hampe dont le diamètre était, au minimum, de $14 \mathrm{~mm}$ - ce qui renvoie clairement à un projectile de type sagaie. De même, parmi les 549 pointes à base fourchue d'Isturitz, Gourdan et Lortet, 42 possèdent une largeur proximale supérieure ou égale à $12 \mathrm{~mm}$ (maximum 17), valeur qui paraît beaucoup trop importante pour des armatures de flèches...

On peut donc proposer l'idée que ces différentes armatures ont plutôt été emmanchées sur des sagaies que sur des flèches. Mais cela n'implique pas que tel soit le cas de toutes les pointes à base fourchue magdaléniennes ! La très grande majorité des pointes d'Isturitz, Gourdan et Lortet présentent en effet une largeur de 7 à $10 \mathrm{~mm}$ et une épaisseur de 5 à $8 \mathrm{~mm}$, dimensions compatibles avec un projectile léger tel que ceux tirés à l'arc. De plus les préhampes d'Isturitz, également associées aux pointes à base fourchue, sont de calibre plus modeste que la pièce du Tuc d'Audoubert : 9 à $11 \mathrm{~mm}$ de diamètre maximal. 
Pour tenter d'identifier le mode de propulsion de ces armatures, il faut donc utiliser d'autres méthodes. C'est pourquoi l'ensemble des réflexions développées ici ont été mises à contribution dans le cadre de l'élaboration d'un protocole d'archéologie expérimentale. Cette expérimentation consistait à tirer des pointes à base fourchue en bois de renne dans des carcasses animales, à l'arc et au propulseur, dans l'espoir d'obtenir des fractures d'impact caractéristiques. Les tirs ont eu lieu au CEDARC/musée du Malgré-Tout (Treignes, Belgique) les 11 et 12 janvier 2003. Les premiers résultats en ont été présentés récemment (Pétillon, sous presse) : ils vont dans le sens de l'identification de l'ensemble des pointes à base fourchue comme armatures de sagaie, sur la base de la morphologie de leurs fractures. Ces données sont cependant encore en cours d'analyse, et de nouvelles expérimentations seront certainement nécessaires pour affiner nos interprétations.

\section{Remerciements}

Nos plus vifs remerciements à Christiane Leroy-Prost, qui nous a signalé la présence de ces deux pièces au Musée de l'Homme et nous en a proposé l'étude ; merci également à Aline Averbouh, Robert Begouën, Jean Clottes, Valérie Feruglio et Carole Fritz pour leurs informations sur la grotte du Tuc d'Audoubert.

\section{Notes}

(1) Notons au passage que si la similarité typologique des pointes à base fourchue dans les quatre sites ne fait pas de doute, leur situation chronologique relative est plus incertaine. La date du Tuc d'Audoubert se place ainsi parmi les dates anciennes du Magdalénien moyen pyrénéen, mais nous verrons plus loin que son association avec les deux pièces étudiées ici n'est pas évidente, celles-ci ayant été découvertes à l'écart du gisement. Les pointes à base fourchue d'Isturitz proviennent quant à elles de couches attribuées au Magdalénien supérieur, mais ces couches n'ont jamais fait l'objet de datations radiométriques. Enfin à Gourdan et Lortet, l'absence de toute stratigraphie précise, due à l'ancienneté des fouilles (années 1870), empêche de préciser la position chronologique des pointes à base fourchue à l'intérieur du Magdalénien...

(2) Certains éléments intermédiaires de hampe de projectile, s'ils correspondent bien à cette définition, ne sont cependant pas des "préhampes" car ils ne se situent pas immédiatement derrière la pointe mais plus bas sur le fût. On trouve ainsi en ethnographie inuit des sagaies dont la pointe et la hampe sont entièrement composées de segments en bois de renne fixés les uns aux autres (Cattelain et Bellier, 2002, fig. 17, $\mathrm{n}^{\circ}$ 15).

(3) "...dans un des niveaux du Magdalénien moyen fut trouvée la longue pointe de flèche représentée sur la figure 16 ; en la lavant dans le ruisseau voisin et en la nettoyant, nous nous sommes aperçus qu'elle se composait de deux moitiés qui s'emboîtaient parfaitement l'une dans l'autre..." (trad. des auteurs).

\section{Références bibliographiques}

AVERBOUH A. (2000) - Technologie de la matière osseuse travaillée et implications palethnologiques, mémoire de doctorat de l'Université Paris I, 2 vol., 500 p.

BÉGOUËN H. (1926) - L'art mobilier dans la caverne du Tuc d'Audoubert (Ariège), IPEK, 1926, p. 219-228. 
BÉGOUËN H., BREUIL H. (1958) - Les Cavernes du Volp. Trois-frères, Tuc d'Audoubert, à Montesquieu-Avantès (Ariège), Paris, Arts et métiers graphiques, 124 p.

BÉGOUËN R., CLOTTES J. (1983) - A propos d'une datation radiocarbone de l'habitat magdalénien du Tuc d'Audoubert, Bulletin de la société préhistorique de l'Ariège, t. XXXVIII, p. 119-122.

BÉGOUËN R., CLOTTES J. (1990) - Art mobilier et art pariétal dans les cavernes du Volp, in J. Clottes dir., L'art des objets au Paléolithique, $t$. 1 : L'art mobilier et son contexte, colloque de Foix - Le Mas d'Azil (1987), actes des colloques de la Direction du Patrimoine, p. 157-172.

BERGMAN C.A. (1987) - Hafting and use of bone and antler points from Ksar Akil, Lebanon, in D.Stordeur dir., La main et l'outil - manches et emmanchements préhistoriques, colloque de Lyon (1984), Paris, CNRS (Travaux de la Maison de l'Orient n 15), p. 117-126.

CATTELAIN P. (1993) - Fiche éléments intermédiaires de hampes de projectiles, in Commission de nomenclature sur l'industrie de l'os préhistorique, Fiches typologiques de l'industrie osseuse préhistorique - cahier 6 : éléments récepteurs, Treignes, CEDARC, p. 15-22.

CATTELAIN P. (1994) - La chasse au Paléolithique supérieur : arc ou propulseur, ou les deux ? Archéo-Situla, t. 21-24, p. 5-26.

CATTELAIN P., BELliER C. (2002) - La Chasse dans la Préhistoire du Paléolithique au Néolithique en Europe... et ailleurs, catalogue d'exposition, Treignes, CEDARC, 70 p.

HERNÁNDEZ PACHECO E. (1923) - La Vida de nuestros antecesores paleolíticos, Madrid, Museo nacional de ciencias naturales, $38 \mathrm{p}$.

PASSEMARD E. (1917) - Sur les pointes de sagaies fourchues, Bulletin de la société préhistorique française, t. 14, p. 119-126.

PÉTILLON J.M. (2000a) - Les Pointes à base fourchue magdaléniennes : problèmes typologiques, techniques et fonctionnels, mémoire de DEA de l'Université Paris I, 57 p.

PÉTILLON J.M. (2000b) - Les pointes à base fourchue magdaléniennes : approche fonctionnelle, Préhistoire et anthropologie méditerranéennes, t. 9, p. 29-55.

PÉTILLON J.M. (2002) - Typologie et utilisation : l'exemple des pointes à base fourchue magdaléniennes, in Patou-Mathis M., Cattelain P. et Ramseyer D. (coord.), L'industrie osseuse préet protohistorique en Europe. Approches technologiques et fonctionnelles. Actes du colloque 1.6, XIVe Congrès de l'UISPP, Liège, 2-8 septembre 2001. Bulletin du Cercle Archéologique HesbayeCondroz, t. XXVI, 105 pages.

PÉTILLON J.M. (sous presse) - Tir expérimental de pointes à base fourchue en bois de renne. in Industrie osseuse et parures du Solutréen au Magdalénien en Europe. Actes de la table ronde sur le Paléolithique supérieur récent, Angoulême, 28-30 mars 2003.

SPEISER F. (1909) - Pfeile von Santa-Cruz, Archiv für Anthropologie, n.s. vol. 8, p. 308-311. 


\section{$\underline{\text { Légendes des figures }}$}

Figure 1 : Plan partiel de la grotte du Tuc d'Audoubert. La galerie fouillée par H. Bégouën figure en noir ; elle communique au nord avec la Salle Nuptiale (niveau médian) et au sud avec la galerie inférieure. (d'après Bégouën et Breuil, 1958 ; modifié par R. Bégouën).

Figure 2 : Pointe à base fourchue du Tuc d'Audoubert $\left(\mathrm{n}^{\circ} \mathrm{MH} 55.33 .9 .2\right.$; dessin R. et J.M. Pétillon).

Figure 3 : Largeur et épaisseur des pointes à base fourchue d'Isturitz, Gourdan et Lortet. La pointe à base fourchue du Tuc d'Audoubert est représentée par un point noir.

Figure 4 : Préhampe du Tuc d'Audoubert (nº MH 55.33.9.1; dessin R. et J.M. Pétillon).

Figure 5 : Proposition de restitution de l'extrémité distale de la préhampe (dessin J.M. Pétillon).

Figure 6 : Détail de l'emboîtement de la pointe à l'extrémité de la préhampe (moulage réalisé par le musée de l'Homme ; photo J.M. Pétillon). 


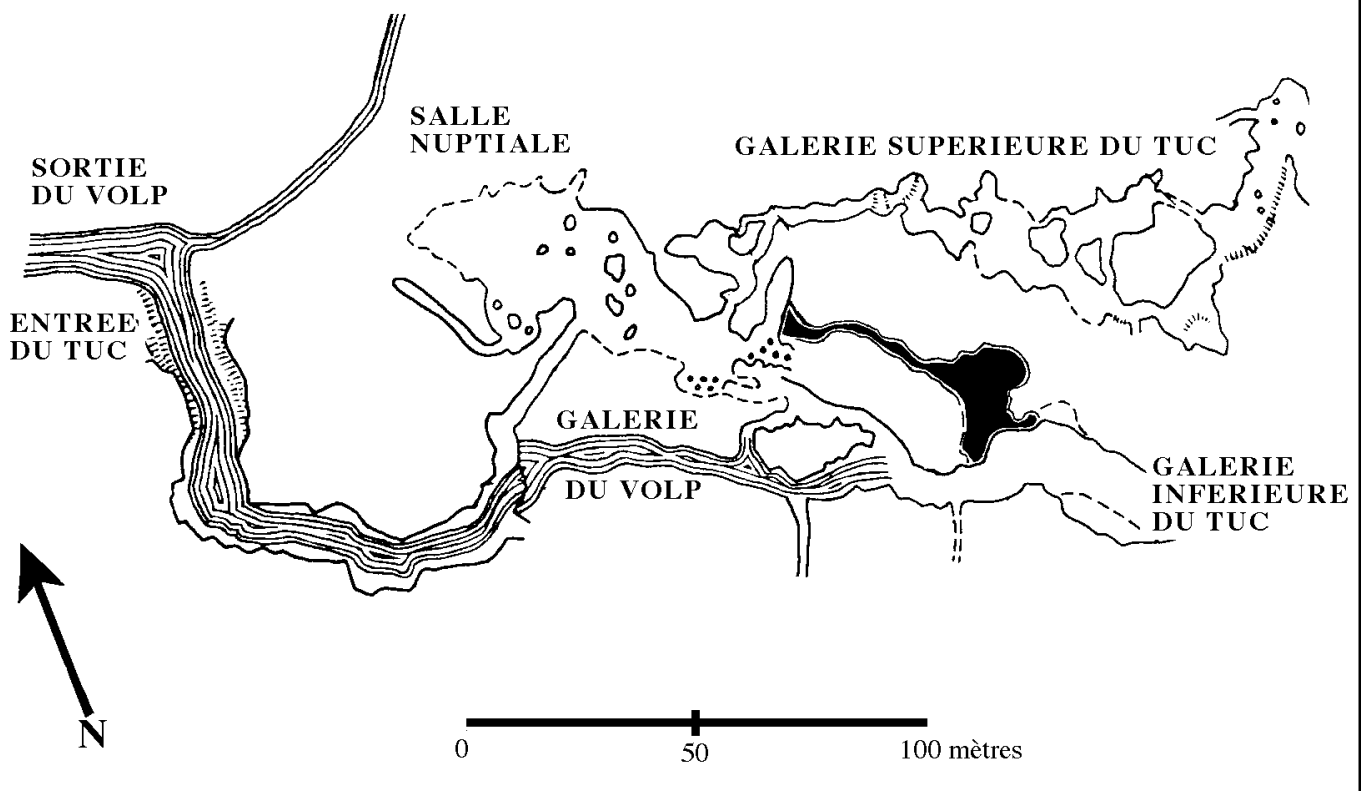



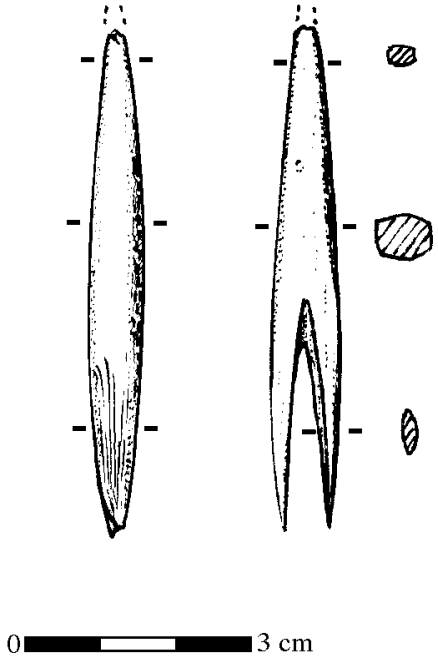


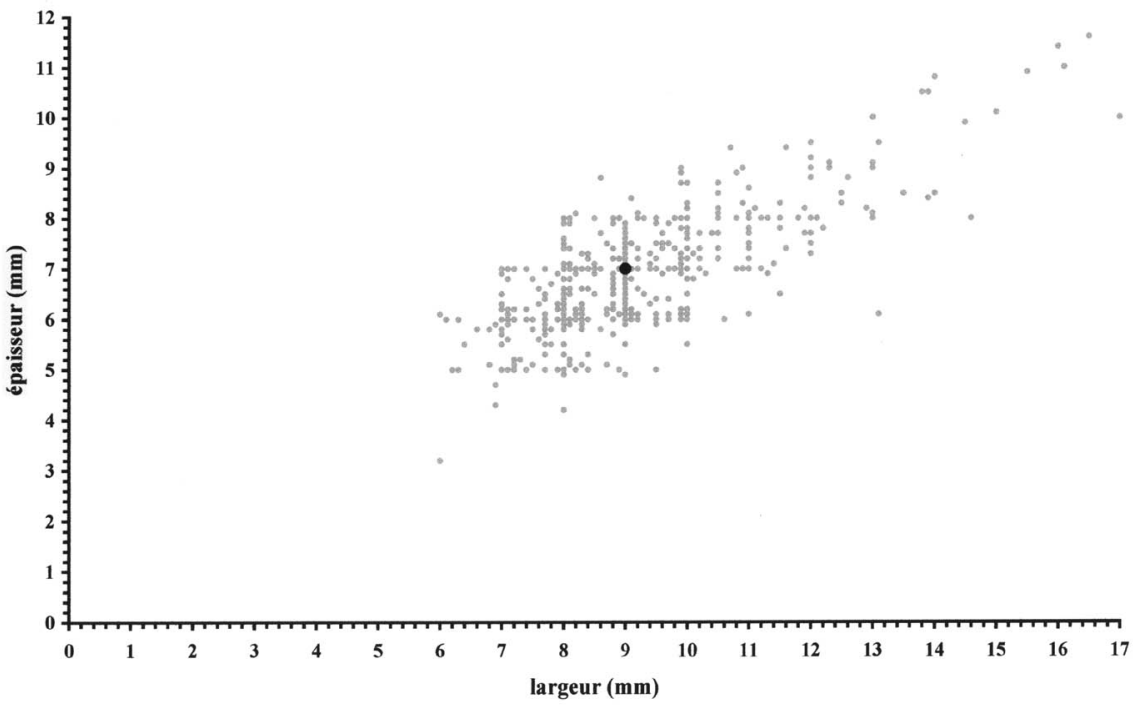




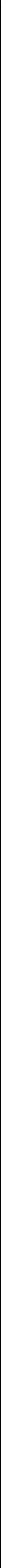




$$
11
$$




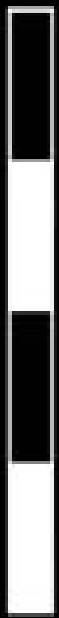




\begin{tabular}{|l|c|c|c|c|}
\cline { 2 - 5 } \multicolumn{1}{c|}{} & $\begin{array}{c}\text { Tuc d'Audoub. } \\
\text { (1 pointe) }\end{array}$ & $\begin{array}{c}\text { Isturitz } \\
(371 \text { pointes })\end{array}$ & $\begin{array}{c}\text { Gourdan } \\
(106 \text { pointes })\end{array}$ & $\begin{array}{c}\text { Lortet } \\
(72 \text { pointes })\end{array}$ \\
\hline épaisseur maximum & 7 & 7,1 & 6,5 & 6,5 \\
\hline largeur maximum & 9 & 9,3 & 8,8 & 8,8 \\
\hline longueur partie prox. & 32 & 32,7 & 32,6 & 31,1 \\
\hline écartement fourche & 5,5 & 4,8 & 4,1 & 4,8 \\
\hline
\end{tabular}

Tableau 1 : comparaison des dimensions moyennes des pointes à base fourchue du Tuc d'Audoubert, d'Isturitz, de Gourdan et de Lortet (mesures en mm) 


\begin{tabular}{|l|c|c|}
\cline { 2 - 3 } \multicolumn{1}{c|}{} & $\begin{array}{c}\text { Tuc d'Audoub. } \\
(1 \text { préhampe })\end{array}$ & $\begin{array}{c}\text { Isturitz } \\
(26 \text { préhampes })\end{array}$ \\
\hline largeur extrémité fourchue & 9 & 9,3 \\
\hline longueur partie rainurée & 22 & 19,9 \\
\hline longueur totale fourche & $25+(*)$ & 30,5 \\
\hline
\end{tabular}

Tableau 2 : comparaison des dimensions moyennes de l'extrémité fourchue des préhampes d'Isturitz et du Tuc d'Audoubert (mesures en mm)

$(*)$ la longueur est incomplète puisque la pièce est fracturée. La proposition de reconstitution (fig. 4) donne une longueur de $31 \mathrm{~mm}$. 